\title{
LO PÚBLICO Y LO PRIVADO EN LOS ESCRITOS DE ALEXANDER VON HUMBOLDT SOBRE CUBA*
}

\author{
Sandra Rebok \\ Depto. Historia de la Ciencia, Instituto de Historia, CSIC
}

\section{RESUMEN}

Este trabajo tiene el objeto de recopilar los comentarios y juicios que hace Humboldt sobre Cuba y valorar su carácter. El análisis e interpretación que resultará de ello se realiza en dos niveles distintos: uno se refiere a las informaciones que se encuentran en escritos no oficiales, es decir, en las cartas que Humboldt envió durante o poco tiempo después de sus visitas a Cuba, y en los diarios que utilizó a lo largo de su expedición. El otro se fundamenta en los escritos oficiales, que comprenden su narración del viaje, el famoso Ensayo sobre Cuba y la memoria autobiográfica escrita en 1804. Mediante el contraste de estas descripciones se pretende comparar las apreciaciones de este viajero prusiano elaboradas en el preciso momento que visita la isla, cotejándolas con las obras que redactó 22 años después de su vuelta a Europa.

PALABRAS CLAVE: Alexander von Humboldt, Cuba, obras publicadas, diarios, correspondencia.

\section{SUMMARY}

This study seeks to collect the comments and judgements that Humboldt makes about Cuba and evaluate their character. The resulting analysis and interpretation will be undertaken on two different levels: One refers to the information that can be found in his unofficial works, which means the letters Humboldt sent during or shortly after of his visits to Cuba, as well as the diaries he used during his expedition. The other is based on the official works, which include his travel description, his famous Essay on Cuba and his autobiographical text written in 1804. Constrasting these descriptions, we will compare the evaluations of this Prussian traveller elaborated in the moment he visited the island, with the works he wrote 22 years after his return to Europe.

KEY WORDS: Alexander von Humboldt, Cuba, published works, diary, correspondence.

* Trabajo realizado con una beca I3P del CSIC y en el marco del proyecto de investigación del Ministerio de Educación y Ciencia, número BHA 2003-04414-C03-01, que dirige el Dr. Miguel Ángel Puig-Samper. Ya fueron publicadas dos versiones preliminares de este estudio: «Alejandro de Humboldt en Cuba: reflexiones historiográficas». En: OPATRNÝ, J. (ed.), (2001), El Caribe Hispano. Sujeto y objeto en política internacional, Praga, Universidad Carolina de Praga, Editorial Karolinum. «Nuevas perspectivas sobre la estancia de Alexander von Humboldt en Cuba». En: Lohania Aruca, Reinaldo Funes y Roberto Díaz (eds.), Expediciones, exploraciones y viajeros en el Caribe. La Real Comisión Guantánamo en la isla de Cuba, 1797-1802. La Habana, Ediciones UNION, pp. 52-66. 
El viajero y científico Alexander von Humboldt estuvo en dos ocasiones en Cuba durante su famosa expedición americana realizada entre 1799 y 1804. La primera estancia abarcó desde el 19 de diciembre de 1800 hasta el 15 de marzo de 1801, cuando decidió abandonar la isla antes de lo previsto con el objeto de incorporarse en Lima a una expedición alrededor del mundo, encabezada por el capitán francés Thomas Nicolas Baudin ${ }^{1}$. Casi al final de su viaje, del 19 de marzo de 1804 al 29 de abril del mismo año —entre su larga estancia en México y su visita a los Estados Unidos- se produjo su segunda estancia en la mayor de las Antillas, principalmente para recoger los materiales que él y su compañero de viaje Bonpland, habían dejado años antes al cuidado del químico cubano Francisco Remírez.

Estas dos estancias del célebre viajero en Cuba, por lo regular han sido tratadas de modo superficial por la bibliografía humboldtiana, ya que, comparado con otros lugares visitados por Humboldt, es relativamente poco lo que se conoce sobre sus actividades en la isla: investigaciones científicas, recorrido, contactos, etc. Aunque le dedicó uno de sus dos tratados socio-politicoeconómicos, el Ensayo político sobre la Isla de Cuba, Humboldt dejó pocas descripciones acerca de su visita a esta isla. No disponemos de una versión continua y completa acerca del tiempo que pasó en Cuba, sino solamente comentarios sueltos que se encuentran en sus diarios y cartas, algunas descripciones de su narración del viaje, la Relation historique, e informaciones dispersas en el Ensayo. Aparte de su estudio de la realidad política, económica y social de Cuba reflejado en este Ensayo, Humboldt incorporó resultados de sus investigaciones sobre la isla en otras obras suyas 2 : en Recueil des $o b$ servations astronomiques ${ }^{3}$ publicó las posiciones de los lugares medidos en Cuba, en Nova Genera et species plantarum ${ }^{4}$ encontramos descripciones y dibujos de las plantas que él y Bonpland hallaron en esta isla, y en el Examen

1 Según las informaciones que Humboldt había recibido, en este momento Baudin haría escala por este puerto- una información que resultó errónea, como Humboldt podría comprobar posteriormente.

2 Estas obras no serán incluidas en el presente análisis.

3 HumboldT, A. de (1808-1810), Recueil d'observations astronomiques, d'opérations trigonométriques et de mesures barométriques, faites pendant le cours d'un voyage aux régions équinoxiales du Nouveau Continent, depuis 1799 jusqu'en 1803, Rédigé et calculé par J. Oltmanns. Vol. 1-2, Paris.

4 Humboldt, A. von, BonPland, A. y Kunth, C.S. (1815-25), Nova genera et species plantarum, Vol. 1-7. Lutitiae Parisioru. 
critique de l'histoire de la géographie du Nouveau Continent ${ }^{5}$ se ocupa de la historia del descubrimiento de Cuba.

Este trabajo tiene el objeto de recopilar los comentarios y juicios que hace Humboldt sobre Cuba y valorar su carácter. El análisis e interpretación que resultará de ello se realiza en dos niveles distintos: uno se refiere a las informaciones que se encuentran en escritos no oficiales, es decir, en las cartas que Humboldt envió durante o poco tiempo después de sus visitas a Cuba, y en los diarios que utilizó durante su expedición. El otro se fundamenta en los escritos oficiales, que comprenden su narración del viaje, su tratado sobre Cuba, el ya mencionado Ensayo y la memoria autobiográfica escrita en $1804^{6}$. Mediante el contraste de estas descripciones se pretende comparar las apreciaciones de este viajero prusiano elaboradas en el preciso momento que visita la isla, cotejándolas con las obras que redactó 22 años después de su vuelta a Europa, y que contienen valoraciones que pueden haber surgido posteriormente, influidas y modificadas por diferentes circunstancias.

En este tipo de análisis, dentro de ese contexto, la inclusión de los diarios de Humboldt tiene un carácter novedoso. Fue el Centro de Investigación Alexander von Humboldt de la Academia de Ciencias de Berlín - a cargo de Margot Faak - quien comenzó en el año 1982 la edición de estos apuntes que Humboldt realizó a lo largo de su expedición americana ${ }^{7}$. En 2000 se terminó el último tomo ${ }^{8}$ de esta gran obra que - debido a los criterios de edición seguidos - corresponde cronológicamente a los preparativos y al inicio de su famoso viaje. Como hasta ahora en su totalidad se han editado sólo en alemán

5 Humboldt, A. de (1836-39), Examen critique de l'histoire de la géographie du Nouveau Continent et des progrès de l'astronomie nautique aux quinzième et seizième siècles, Paris, T. 1-5.

6 ReboK S. y Puig-SAmPer, M.A. (2002), «Alexander von Humboldt y el relato de su viaje americano redactado en Filadelfia», Revista de Indias, 224 (LXI), 209-223.

7 FAAK, M. (edit.) (1982), Lateinamerika am Vorabend der Unabhängigkeitsrevolution. Eine Anthologie von Impressionen und Urteilen aus den Reisetagebüchern, Berlin, AkademieVerlag, Tomo 5; (1986), Alexander von Humboldt. Reise auf dem Rio Magdalena, durch die Anden und durch Mexiko, Berlin, Akademie-Verlag, Tomo 8; (1990), Alexander von Humboldt. Reise auf dem Rio Magdalena, durch die Anden und durch Mexiko, Berlin, AkademieVerlag, Tomo 9. FAAK, M. (edit.) (2000), Alexander von Humboldt. Reise durch Venezuela, Berlin, Akademie-Verlag, Tomo 12.

8 Véase: PuIG-SAMPER, M.Á. y REBOK, S. (2001), Reseña del libro Reise durch Venezuela. Auswahl aus den amerikanischen Reisetagebüchern (editado por Margot Faak), Revista de Indias, 223, 773-775. 
—y en francés las partes que redactó en este idioma una barrera que dificulta la integración de estos valiosos documentos a los estudios que se realizan en la actualidad. Sin duda, los diarios de Humboldt —en primer lugar por ofrecernos una impresión detallada de su viaje en el mismo momento en que se produce, así como sus primeros juicios, comentarios y vivencias, sin posteriores reflexiones o auto-censuras - constituyen un fundamento importantísimo para la investigación humboldtiana en general, y en particular para Cuba, pues incluyen aspectos novedosos acerca de esta isla.

\section{COMENTARIOS DE HUMBOLDT ACERCA DE CUBA Y SUS ESTANCIAS ALLÍ}

La gran obra del viaje americano de Humboldt, Voyage aux régions équinoxiales du Nouveau Continent ${ }^{10}$, consta de 30 tomos que tratan aspectos diversos de esta expedición; algunos de ellos fueron publicados más tarde como libros separados. Contiene también su famosa narración del viaje, llamada Relation historique, cuyo capítulo XXVII comprende el trayecto de Humboldt desde la costa de Venezuela a La Habana, con informaciones generales sobre la población de las Antillas, comparadas con la población del Nuevo Continente, bajo diferentes aspectos como la diversidad de razas, la libertad personal, los idiomas y las culturas.

En el capítulo XXVIII nos encontramos el Ensayo político sobre la isla de $C u b a$, con sus subdivisiones alrededor de diferentes temas y su último apartado donde retoma la narración de su viaje describiendo su exploración por el Valle de Güines, Batabanó y puerto de Trinidad.

A la travesía que realiza desde Trinidad al Río Sinú y a Cartagena de Indias, así como a los primeros momentos de su estancia en la actual Colombia,

9 Sólo se han editado ediciones parciales de lo referente a Colombia, Perú y México: Humboldt, A. von (1982), Extractos de su diarios/Auswahl aus seinen Tagebüchern, Bogotá, Publicismo y Ediciones; (1991), Humboldt en el Perú. Diario de Alejandro de Humboldt durante su permanencia en el Perú (agosto a diciembre de 1802), Piura, Centro de Investigación y Promoción del Campesinado (CIPCA). «Diario de viaje (de Acapulco a Veracruz, 1803-1804)», publicado en: Humboldt, Alexander von, Tablas geográficas políticas del Reyno de Nueva España. México: siglo xxi editores, 2003 (coordinadores: Charles Minguet y Jaime Labastida), pp. 215-311.

10 Humboldt, A. y BonPland, A. (1816-1831), Voyage aux régions équinoxiales du Nouveau Continent, fait en 1799, 1800, 1801, 1802, 1803 et 1804 par A. de Humboldt et A. Bonpland, Réd. Par A. de Humboldt avec un atlas géographique et physique. 13 tomos. Paris, Librairie greco-latine-allemande. 
dedica el siguiente capítulo. Aquí finaliza la narración de su viaje, por lo tanto no nos da ninguna información sobre su segundo viaje a la isla caribeña. En lo que se refiere a ella, vemos que Humboldt concede mucho espacio a la descripción de la llegada y salida de la isla, dedicando un capítulo completo a cada una. Pero respecto a sus estancias en Cuba - quitando el Ensayo del que hablaremos a continuación - no nos cuenta prácticamente nada en esta narración del viaje.

\section{I.1. Ensayo político sobre la isla de Cuba}

Lo que entendemos hoy por el Ensayo político sobre la Isla de Cuba ${ }^{11}$ está compuesto de tres partes diferentes: el texto del Ensayo mismo de 1826, el Análisis racionalizado del mapa de la Isla de Cuba del mismo año y el Cuadro estadístico de la isla de Cuba para los años 1825 y 1829. Las valoraciones y los datos proceden del conocimiento directo de Humboldt, que son el resultado de experimentos realizados, como la medición de la posición de La Habana, recolección de plantas, un análisis del agua y la temperatura, etc., o de la recopilación de numerosos datos y ciertos documentos proporcionados por la élite científica y social de Cuba, así como su propia capacidad de análisis.

El panorama histórico-cultural y socio-político de la isla de Cuba que Humboldt nos ofrece fue elaborado basándose en informaciones recibidas después de su estancia y publicado en 1826, es decir, 22 años después de su última estancia en Cuba. En todo este tiempo, Humboldt se mantuvo en contacto con sus amigos cubanos, recopiló informaciones, analizó hechos, estableció relaciones. Como resultado de ello, su libro al publicarse estaba al día y reflejaba la realidad contemporánea de la isla. Como contraste a esta recopilación minuciosa de datos valiosos sobre el país y las diferentes facetas de su realidad - economía, sociedad, agricultura etc.--, debido quizá al carácter de este tratado, el Ensayo apenas contiene informaciones sobre su estancia, viajes por Cuba, actividades o experiencias. Sólo en el último capítulo de esta obra, titulado Viaje al valle de Güines, al Batabanó y al puerto de la Trini-

11 Para informaciones detalladas acerca de las diferentes ediciones, las traducciones y la historia de su recepción, véase: BECK, H. (ed.) (1992), Alexander von Humboldt. Cuba-Werk, Darmstadt, Wissenschaftliche Buchgesellschaft, pp. 229-264; PRÜFER-LESKE, Irene, «Übersetzungen, Manipulation und Neuübersetzung des Essai politique sur l'île de Cuba Alexandere von Humboldts» en ETTE O., BERNECKER W. L. (eds.) (2001), Ansichten Amerikas. Neuere Studien zu Alexander von Humboldt, Frankfurt a.M., Vervuelt Verlag, pp. 219-230. 
dad, y a los jardines y jardinillos del rey y de la reina, que redactó en parte en forma de relato de viaje, el científico prusiano da informaciones sobre sus actividades en la isla. Entre las pocas reseñas que ofrece respecto a este tema hay, sobre todo, descripciones de tipo muy general o comentarios sueltos que reflejan una impresión momentánea. Así comenta que:

«Dos veces he estado en la isla, la una tres meses, y la otra mes y medio, y he tenido la fortuna de gozar la confianza de personas que, por sus talentos y por su situación, como administradores, propietarios, comerciantes, podían darme noticias acerca del aumento de la prosperidad pública. Esta confianza era muy legítima por la protección particular con que me ha honrado el ministerio español; y me lisonjeó también de haberla merecido, por la moderación de mis principios, por una conducta circunspecta y por las clases de mis pacíficas ocupaciones» ${ }^{12}$.

Sobre su alojamiento dice:

«Hallamos en la familia de Cuesta, que con la de Santamaría formaba una de las mayores casas de comercio de la América, y en la casa del Conde de O'Reilly, la hospitalidad más noble y generosa. Nos alojamos en casa del primero y pusimos nuestros instrumentos y nuestras colecciones en el vasto palacio del conde, cuyas azoteas eran particularmente a propósito para las observaciones astronómicas» ${ }^{13}$.

Además de estas informaciones de carácter general, encontramos comentarios ambiguos de Humboldt acerca de su estancia en Cuba, lo que a primera vista puede crear dudas sobre su verdadera postura respecto a ésta. Tales contrastes no se encuentran sólo comparando sus escritos oficiales con los no oficiales, sino también en los propios textos. Esto se observa, por ejemplo, en un comentario - en este caso positivo - que hace sobre La Habana, al momento de llegar al puerto:

«No he recorrido conjuntamente con Bonpland, sino las cercanías de la Habana, el hermoso valle de Güines, y la costa entre el Batabanó y el puerto de la Trinidad. (...) La vista de la Habana, a la entrada del puerto, es una de las más alegres y pintorescas de que puede gozarse en el litoral de la América equinoccial, al norte del ecuador. Aquel sitio, celebrado por los viajeros de todas las naciones, no tiene

12 Humboldt, A. von (1998), Ensayo político sobre la isla de Cuba, Aranjuez, Ediciones Doce Calles, Junta de Castilla-León. (Estudio introductorio y edición de Miguel Ángel Puig-Samper, Consuelo Naranjo Orovio y Armando García González). THEATRUM NATURAE. Colección de Historia Natural, Textos Clásicos), p. 105.

13 Ibidem, p. 120. 
el lujo de vegetación que hermosea las orillas del Guayaquil, ni la majestad de silvestre de las costas rocallosas del Río Janeiro (...), pero la gracia que en nuestros climas adorna las escenas de la naturaleza cultivada, se mezcla allí con la majestad de las formas vegetales, y con el vigor orgánico característico de la zona tórrida» ${ }^{14}$.

Sin embargo, después de estos elogios, en la siguiente página nos ofrece una descripción bastante negativa de la ciudad de La Habana:

«Durante mi mansión en la América española, pocas ciudades de ella presentaban un aspecto más asqueroso que la Habana, por falta de una buena policia; porque se andaba en el barro hasta la rodilla; y la muchedumbre de calesas o volantes, que son los carruajes de la Habana; los carros cargados de cañas de azúcar, y los conductores que daban codazos a los transeúntes, hagan enfadosa y humillante la situación de los de a pie» ${ }^{15}$.

Tales juicios no tienen que ser considerados contradictorios, pero ya que constrastan considerablemente, estas visiones de La Habana hacen pensar que su primera impresión de Cuba fue ambigua. También se pueden explicar estos notables contrastes por la tendencia de Humboldt de describir la naturaleza americana en toda su grandiosidad, concediéndole un cierto protagonismo en sus reflexiones y valoraciones. Lo humano, al contrario, y con ello las ciudades, ocupan un lugar menor y son descritos con menos entusiasmo y más crítica. Ello se puede aplicar en el ejemplo supracitado, la primera cita se refiere más a la naturaleza alrededor de La Habana, mientras la segunda refleja su impresión de la ciudad como tal ${ }^{16}$.

El juicio de Humboldt sobre las mujeres cubanas, resulta, no obstante, siempre igual:

«Nos admiraron de nuevo la alegría y viveza de ingenio de las mujeres de $\mathrm{Cu}$ ba, igualmente en la provincia que en la capital. Son unos dones felices de la naturaleza a los que el refinamiento de la civilización europea puede dar más atractivo; pero que agradan ya en su sencillez primitiva $\gg^{17}$.

14 Ibidem, pp. 107.

15 Ibidem, p. 108.

16 Este contraste entre las descripciones que Humboldt hace de la naturaleza y las ciudades americanas lo encontramos descrito con más ejemplos en LUCENA, M. (2002), «Alejandro de Humboldt y la invención del Trópico», en Gomez, Th. (ed.) (2002), Humboldt et le monde hispanique, París/Nanterre, Centre de recherches Ibériques et Ibéro-américaines, pp. 43-57.

17 Humboldt (1998), p. 344. 
El Ensayo está en la línea de las obras de Humboldt - es muy completo en los aspectos tratados, contiene informaciones variadas y muestra el enfoque holístico que caracteriza sus investigaciones - , pero la parquedad de descripción de su estancia nos da la idea de que fue, para él, menos significativo el tiempo que pasó en Cuba que la elaboración de datos e informaciones recogidas posteriormente.

\section{I.2 Diarios}

Tampoco es posible realizar, siguiendo los diarios que se conservan, una reconstrucción de las visitas de Humboldt a Cuba, ya que no existe en ellos - ni para su primera, ni para su segunda estancia - una narración continua y detallada. Lo que sí encontramos son bastante más descripciones sobre su llegada y salida en barco que su estancia en tierra cubana, así como comentarios dispersos que se hallan en capítulos referentes a otras regiones.

Antes de analizar las ideas que Humboldt vierte en sus diarios sobre Cuba, se precisa una observación general sobre el carácter de éstos. No constaban de un sólo libro donde apuntase de manera sucesiva sus observaciones e impresiones, sino de diferentes cuadernos para diversas disciplinas, distinguidas por sus colores. A su vuelta a Europa deshizo estos cuadernos a fin de ponerlos a disposición de sus colaboradores, lo que hizo difícil la reconstrucción de los diarios ${ }^{18}$; es posible, además, que se hayan perdido parte de esos cuadernos. Por lo tanto, Humboldt pudo haber hecho descripciones sobre Cuba que no se han podido encontrar e incluir en la edición de sus diarios.

Lo que hallamos es una descripción muy extensa de su viaje de Nueva Barcelona a La Habana (del 24 de noviembre al 19 de diciembre de 1800) - que abarca 30 páginas en la edición de los diarios de Margot Faak ${ }^{19}$-, con mediciones y observaciones de todo tipo, ilustradas con diversos dibujos, que termina con la llegada de Humboldt a Cuba en la mañana del día 19; así como una narración de su estancia en la isla desde el momento que salió de La Habana el 5 de marzo del año siguiente - tras haber estado allí durante dos meses y medio - para embarcarse cuatro días después en Batabanó rumbo a Cartage$\mathrm{na}^{20}$. Pero sobre el tiempo que pasó en La Habana y excursiones a sus alrededores, no comenta nada.

18 FAAK (2000), p. 18.

19 Ibidem, pp. 393-422.

20 FAAK (1986), pp. 41-48. 
Comienza su descripción con comentarios sorprendentemente negativos, llamando Batabanó «un pueblecito miserable, que sólo desde hace pocos años tiene una iglesia» ${ }^{21}$. La Ciénaga, al sur de Batabanó, la describe como «una región terriblemente pantanosa, que sólo tiene hierbas, iridoidea y palmeras de abanico enfermizas ${ }^{22}$. Sigue con diversas observaciones de carácter geológico y mineralógico, reflexiones sobre la existencia de agua dulce en la isla, estudios sobre la temperatura y la composición del agua del mar, y comentarios sobre los cayos; incluye también algunos juicios generales - y algo negativos- sobre la poca población de Cuba y su carácter desierto. Interesante es la referencia a una invitación que tuvieron él y Bonpland en Trinidad en casa del teniente gobernador Vicente y Ulloa, un sobrino del celebre astrónomo don Antonio Ulloa. Vicente les preparó:

«un gran convite con comida indigerible, emigrados más indigeribles todavía que se anidaron por todas las partes, y versos horribles, que un doctor teologíae obeso (que a pesar del terrible calor del día estaba vestido de terciopelo) recitaba para elogio mío con una lentitud embarazosa. El teniente gobernador (apenas 180 pesos de ingresos, aunque gobernador de 4 pueblos, Trinidad, Villa Santa Clara, El Puerto Príncipe, y Sancti Spiritus) me dijo de manera muy ingenua, que para sentir vivamente como era el tono de esta ciudad, solo tenía que mirar alrededor de mí; el doctor es un cura malísimo y el más erudito de todos... En la casa de Don Antonio Padrón, la familia más rica, mucha compañía de mujeres, viejas hermanas muy vivas, algo impertinentes. Pero todo demostraba que uno se encontraba en la isla de Cuba. Las mujeres de esta isla tienen una vivacidad, una agilidad, por lo que se distinguen con gran distancia de las criaturas insignificantes e indolentes de los indios de Caracas y Cumaná» ${ }^{23}$.

21 Ibidem, p. 41. En el original: «Batabanó, ein elendes Dörfchen, das erst seit wenigen Jahren eine Kirche hat». (traducción propia)

22 Idem. En el original: (...) «eine fürchterlich morastige Gegend, die bloss Gräser, Irdoidea und kränkliche Fächerpalmen hat» (traducción propia).

23 Ibidem, p. 45. En el original: (...) «ein grosses Festín mit unverdaulichem Essen, noch unverdaulicheren, sich überall einnistenden Emigrierten und fürchterlichen Versen, die ein feister (trotz der schrecklichen Tageshitze in Samt gekleideter) Doctor theologiae zu meinem Lobe mit peinlicher Langsamkeit deklamierte. Der Teniente Gobernador (...) sagte mir sehr naiv, dass, um lebhaft zu fühlen, wie der Ton dieser Stadt sei, ich nur um mich her zu blicken brauche; der Doctos sei ein erzböser Pfaffe und der gelehrteste von allen.... In dem Hause des Don Antonio Padrón, der reichsten Familie, viel Damengesellschaft, sehr lebendige, etwas zudringliche alte Schwestern. Doch alles zeigte, dass man in der Insel Kuba sei. Die Weiber dieser Insel haben eine eigene Lebendigkeit, eine Gewandtheit, die sie meilenweit vor den insignifikanten und indisch indolenten Geschöpfen in Caracas und Cumaná auszeichnet». (traducción propia). 
A continuación describe los dos puertos que tiene Trinidad, las observaciones y mediciones realizadas por él allí, las grandes y rápidas bajadas de temperatura en los llanos de La Habana ${ }^{24}$. Más espacio dedica a los problemas que tiene que enfrentar el resto de Cuba debido al gran tamaño de La Habana y a la corrupción que predomina en esta ciudad, con un gobierno constituido por hacendados y comerciantes. La escasez y la importancia de juicios de Humboldt acerca de estos temas justifica esta cita tan larga:

«La industria azucarera alrededor de Trinidad ha aumentado algo, se transportan anualmente unas 4000 cajas de azúcar; pero la tiranía, que La Habana ejerce sobre el resto de la isla, impide toda industria. Como el gobierno reina desde La Habana, y no ve nunca el resto de la isla, como el consulado está formado por hacendados y comerciantes, cuyo interés es que sólo florezca el comercio de La Habana, no se permite que barcos neutrales entren y carguen aquí, a pesar de que el permiso real incluye todos los puertos. Se pretende que el contrabando sería demasiado grande, como si en alguna parte se pudiese hacer más contrabando de Providence y Jamaica, de lo que hace la clase de hombres más ricos en La Habana desde Batabanó; en La Habana, donde por dinero se pueden conseguir todo tipo de papeles de exportación, certificados como si uno hubiera cargado productos españoles, como si uno tuviera hierro y esclavos, papeles que son transferidos de un barco a otro... Por esto, debido a la falta de recogida, en Trinidad el azúcar no tiene precio; se acumula, mientras el hacendado en La Habana recibe el dinero para sus productos ya antes de cosecharlos. El gran tamaño de La Habana tiene la culpa de que el resto de la isla sea un desierto. Desde Trinidad la desesperación hace que los pobres hacendados en tiempo de paz lleven su azúcar a La Habana a pesar de los gastos de transporte, vía Batabanó por tierra o por el Cabo San Antonio. Esa misma es la situación del Puerto Príncipe, de Santiago de Cuba... Se está pensando, poner inmediatamente una queja en tiempo de paz en Madrid... pero los familiares de los hacendados de La Habana, o sea el partido contrario, son los más ricos, los más prestigiosos, los más hábiles... Aparte de la injusticia es también poco político. El alto precio de todos los alimentos que mata a la industria, el encarecimiento del sueldo, la imposibilidad de defenderse como ciudad militarmente durante mucho tiempo, el lujo, la inmoralidad, la inclinación por novedades impetuosas, la fiebre amarilla - males que proceden una población artificialmente grande, donde dentro y fuera de los muros viven unos 110.000 habitantes, mientras toda la isla tiene 300.000 (entre ellos 50.000 esclavos). Cuantas veces más sabio sería preparar varios puntos centrales en la larga isla.... $\rangle^{25}$

24 Ibidem, pp. 46-47.

25 Ibidem, pp. 47-48. En el original: «Die Zuckerkultur um Trinidad hat etwas zugenommen, man verschifft jährlich ein 4000 Cajas de azúcar; aber die Tyrannei, welche die Havanna auf den Rest der Insel ausübt, hindert alle Industrie. Da die Regierung in Havanna thront, den Rest der Insel nie sieht, da das Consulado aus Hacendados und Kaufleuten besteht, deren 
Respecto a su segunda estancia en Cuba encontramos un corto, pero muy interesante, comentario al principio de su descripción de la salida de $\mathrm{La}$ Habana rumbo a Filadelfia:

«Salimos el 29 de Abril de 1804, todos muy poco contentos con nuestra segunda estancia en La Habana. Este lugar nos parecía agradable en el año 1800, cuando venimos de la soledad del Orinoco. Nos parecía poco interesante, al llegar ahora de México, donde a lo mejor hay algo menos de libertad de pensamiento (si uno puede osar reconocer diferencias más allá del «infinitamente pequeño»), pero por lo menos, importantes instituciones científicas. En La Habana todas las conversaciones giran en torno al gran problema, cómo se puede producir en un día con el menor número de esclavos la cantidad más grande de pilones de azúcar; porque el marqués $\mathrm{X}$, que es favorecido por los comerciantes, vende su azúcar por 17 a 19 pesos, mientras otro solo lo vende por 14 a 16 pesos. De ello resulta nada más que un cuadro de números, no existe ningún interés técnico, ninguna idea física, ninguna investigación de las causas...» ${ }^{26}$

Interesse es ist, dass nur der Handel der Havanna floriere, so erlaubt man nicht, dass neutrale Schiffe hier einlaufen und laden, obgleich die königliche Erlaubnis alle, alle Häfen in sich begreift. Man giebt vor, die Einfuhr der Contrabande würde zu gross sein, eben als ob man irgendwo mehr Contrabande in Providence und Jamaica machen könne, als die reichste Menschenklasse von Havanna und von Batabanó aus betreibt; in der Havanna, wo man für Geld sich jegliche Art von Expeditionspapiere schaffen kann, Zeugnisse, als habe man spanische Produkte geladen, als habe man Eisen und Sklaven; Papiere, die man von einem Schiff auf's andere überträgt... In Trinidad haben daher wegen Mangel der Abnahme Zucker keinen Preis; sie häufen sich an, während dass der Hacendado in der Havanna seine Produkte schon früher bezahlt erhält als er sie einärndtet. Die Grösse Havannas ist schuld, dass die übrige Insel eine Wüste ist. Von Trinidad aus bringt die Verzweiflung die armen Hacendados im Frieden dahin, durch Batabanó zu Lande oder um Cabo San Antonio trotz der Frachtkosten ihren Zucker nach der Havanna zu fahren!! Eben dies ist die Lage des Puerto Principe, von Santiago de Cuba... Man denkt darauf, im Frieden unmittelbar Klage in Madrid zu führen...aber die Verwandten der Hacendados der Havanna, also die Gegenparthei, sind die reichsten, angesehensten, gewandtesten... Ausser der Ungerechtigkeit ist das ganze auch sehr unpolitisch. Die industrietötende Vertheuerung aller Lebensmittel, die Theuerung des Arbeitslohns, die Unmöglichkeit, sich lange als Stadt militärisch zu vertheidigen, Luxus, Sittenlosigkeit, Neigung nach ungesthümen Neuerungen, Gelbes Fieber - alles entstehr von der unnatürlich grossen Population der Havanna, wo in und extra muros ein 110000 Einwohner leben, wenn die ganze Insel nur ein 300000 Einwohner (worunter 50000 Sklaven) hat! Wieviel weiser, mehrere Centralpunkte in der langen Insel anzulegen. (...)» (traducción propia)

26 FAAK (1990), p. 299. En el original: «Wir liefen am 29. April 1804 aus, alle sehr wenig zufrieden mit unserem zweiten Aufenthalt in Havanna. Dieser Ort erschien uns im Jahre 1800 angenehm, als wir aus den Einöden des Orinoco kamen. Er erschien uns wenig interessant, als wir nun aus Mexico anreisten, wo es vielleicht etwas weniger Gedankenfreiheit gibt 
A continuación sigue la extensa descripción del viaje a los Estados Unidos, interrumpida sólo por unas pocas reflexiones de tipo geográfico acerca de Cuba. ${ }^{27}$

En las descripciones de Colombia que realiza en sus diarios incluye reflexiones acerca de Cuba, que se refieren en primer lugar a su administración colonial y las consecuencias sociales de ello.

«De dos a tres grandes haciendas en la isla de Cuba, producen evidentemente tanto azúcar como las muchas familias que viven dispersas en el valle de Guaduas. Pero esas 2 ó 3 haciendas caen en la ruina por el derroche o por la muerte de 2 ó 3 personas. (...) Además, una hacienda de caña, asentada en la isla de Cuba, no produce casi nada excepto azúcar. Sin carne de Barcelona y Buenos Aires muere de hambre la isla de Cuba. Ella depende de factores externos. La familia que cultiva azúcar en pequeñas cantidades, siembra al mismo tiempo su conuco, y se alimenta a sí misma. (...) La isla de Cuba, o más bien, las 100 leguas cuadradas que están sembradas con caña de azúcar, entre La Habana, Matanzas y Batabanó lo mismo que muy cerca de Trinidad y Santiago de Cuba, producen anualmente 3'200.000 arrobas de azúcar (en 1810 la Habana exportó 142.000 cajas de 16 arrobas que valen 40 pesos). La mayor parte de esta monstruosa suma se reparte entre unas 30 familias. Estas viven disipadamente... y la isla es un desierto cubierto de selva desde Batabanó y Matanzas hasta Jagua, Villa Clara y Santiago de Cuba. Qué distinto sería si la Isla Cuba produjera solo $1 / 6$ de los 3 millones de arrobas en pequeñas haciendas de gente libre. Una plantación de café de 60.000 árboles produce un excedente de 25.000 piastras y es trabajada por 40 esclavos. Cuarenta y cien familias podrían vivir holgadamente de este ingreso que ahora despilfarra una sola. Qué diferente sería la población y el cultivo de la isla Cuba. Pero la costumbre vence y la gente está convencida en forma apodíctica que la felicidad de una isla de las Indias Occidentales reside en la cantidad de esclavos y del azúcar que produce, no en la cantidad de brazos libres activos, no en la mayor cantidad de felicidad doméstica de muchos $(\ldots)\rangle^{28}$.

(wenn man es wagen kann, jenseits des „unendlich Kleinen» Unterschiede anzuerkennen), aber wenigstens bedeutende wissenschaftliche Einrichtungen. In Havanna drehen sich alle Gespräche um das grosse Problem, wie man an einem Tage mit der geringsten Zahl von Schwarzen die grösste Menge Zuckerhüte produzieren kann, warum der Marqués X, der von den Kaufleuten begünstigt wird, seinen Zucker zu 17 und 19 Pesos verkauft, während ein anderer ihn nur zu 14 und 16 Pesos verkauft. Daraus resultiert nichts als eine Zahlenübersicht, es gibt kein technisches Interesse, keine physische Idee, keine Untersuchung der Ursachen...» (traducción propia)

27 Ibidem, p. 299.

28 Humboldt (1982), p. 39-40. 
Lo que destaca aquí es que Humboldt no sólo se limita a criticar lo encontrado, sino que basa sus criticas en estudios profundos y propone, además, soluciones que le parecen más humanas.

Como hemos visto, Humboldt nos trasmite en sus diarios una imagen de Cuba, y en particular de La Habana, en parte de carácter negativo. Estos juicios se encuentran enteramente en la línea de su pensamiento y, por lo tanto, parecen reflejar su verdadera imagen de esta isla y su sociedad. Hay que considerar que estos comentarios críticos se refieren a la sociedad colonial de entonces, ya que lo que critica es su administración, el sistema esclavista, la injusticia social, la falta de preocupaciones intelectuales de la élite cubana, las malas condiciones de trabajo de los obreros, etc. Además, especialmente en Cuba, Humboldt se relacionó casi exclusivamente con la élite colonial, moviéndose en el ambiente de los grandes hacendados y azucareros; son éstos los aspectos de la sociedad colonial con los que se enfrenta y los que destaca en sus escritos más privados como son los diarios.

Por razones evidentes, comentarios de este estilo no se hallan en su introducción, ni en su narración del viaje, la Relation historique, ni en su Ensayo sobre Cuba.

Además de estos comentarios, que por su carácter narrativo o descriptivo han sido incluidos en la edición de los diarios realizada por Margot Faak, existen varias hojas con datos sobre Cuba y especialmente La Habana, que no han sido publicadas todavía ${ }^{29}$. Se trata sobre todo de mediciones astronómicas y geográficas de La Habana, San Antonio de los Baños y el cafetal El Fondadero $^{30}$, así como de apuntes suyos donde compara sus propias mediciones de distintos puntos de La Habana con las que habían obtenido otros científicos como Cosme Damián Churruca y Elorza, José Joaquín Ferrer y Cafranga, Dionisio Galiano o también Cristobal Colón ${ }^{31}$. Estos documentos por lo tanto no aportan nada nuevo a la visión humboldtiana respecto a esta isla caribeña, pero sí demuestran su interés científico por llevar a cabo sus habituales mediciones o constrastes también en Cuba.

29 Una lista de todo el material no publicado hasta ahora se encuentra en: FAAK, Margot (2002), Alexander von Humboldts amerikanische Reisejournale. Eine Übersicht. Berliner Manuskripte zur Alexander-von-Humboldt-Forschung, cuaderno 25, Berlin, Alexander-vonHumboldt-Forschungsstelle, 2002.

30 Diario IX, hojas 24V-24R, veáse: Ibidem, p. 33.

31 Diario IX, hojas 114V-115R, véase: Ibidem. p. 34. 


\section{I.3. Cartas}

Al hacer un análisis de los comentarios de Humboldt sobre su estancia en Cuba, que se encuentran en las cartas enviadas desde América, debe recordarse el hecho de que la probabilidad de que tales cartas llegaran a su destino en aquellos tiempos no era tan alta como hoy, y menos todavía en las condiciones y desde los sitios en los que Humboldt enviaba muchas de ellas. Él mismo se queja en varias ocasiones de que una parte de sus cartas se había perdido por el camino. Otro factor es que tampoco se conservan hoy en día todas las cartas recibidas por sus destinatarios, ni se han publicado todas; y, por último, de las que llegaron y se conservaron y fueron publicadas, no tenemos certeza de que estén completas o sólo sean extractos de ellas, o se haya recortado, por el motivo que sea, algún comentario de Humboldt. De ahí, que el análisis de las cartas no pueda ser otra cosa que un simple acercamiento a nuestra pregunta.

Se han podido encontrar sólo dos cartas de las que Humboldt escribió durante su primera estancia en Cuba. Ambas fueron dirigidas al botánico alemán Karl Ludwig Willdenow ${ }^{32}$, pero prácticamente no contienen informaciones sobre sus actividades o contactos en esta isla. En la primera - muy extensadescribe a Willdenow las cajas de plantas que había mandado a Europa, sus planes para la publicación de sus investigaciones, su encuentro con el viajero inglés Fraser y el viaje realizado hasta este momento. La segunda carta parece haber sido bastante corta - si no se trata de un extracto- y contiene solamente la información del envío realizado de una colección de plantas seleccionadas, con algunas instrucciones referentes a ellas. De otra, dirigida a su hermano Wilhelm von Humboldt, sabemos por un comentario que Alexander hace en referencia a ella en su siguiente carta ${ }^{33}$, que no llegó nunca. Como las cartas destinadas a su hermano solían ser más bien largas y con descripciones e informaciones detalladas, es de suponer que esta misiva perdida sí contenía un comentario acerca de su estancia en Cuba.

En la mencionada carta a su hermano enviada desde Cartagena de Indias, Humboldt sólo da una descripción extensa de su salida de Batabanó para Car-

32 Estas cartas, mandadas el 21.2.1801 y el 4.3.1801 desde La Habana, están publicadas en MoheIt, U. (ed.) (1993), Alexander von Humboldt. Briefe aus Amerika 1799-1804, Berlin, Akademie-Verlag, pp. 122-134. La primera está también incluida en su traducción española en Minguet, Ch. (ed.) (1980), Alejandro de Humboldt. Cartas Americanas, Venezuela, Ayacucho, pp. 73-78.

33 Carta enviada el 1 de abril del mismo año desde Cartagena de Indias, en MingueT (1980), p. 78. 
tagena el 8 de marzo y menciona los dos días que pasó en Trinidad. Allí hace énfasis de nuevo en los acontecimientos y actividades ocurridos durante esta navegación. Su extensa descripción incluye sólo dos comentarios bastante positivos acerca del paisaje. Hablando de las cercanías de Trinidad, comenta: «pasamos dos días agradables en una bella y romántica región» ${ }^{34}$.

La siguiente epístola que contiene informaciones sobre su estancia en $\mathrm{Cu}-$ ba es otra dirigida unos meses más tarde a su hermano ${ }^{35}$. En ella Humboldt ofrece un resumen de la parte de la expedición realizada hasta entonces, donde incluye una referencia al tiempo pasado en Cuba.

«Llegamos a La Habana en diciembre, después de una travesía muy tormentosa y muy larga, de un mes y medio, durante la cual casi naufragamos en los arrecifes del banco de la Víbora, al sur de Jamaica. Allí pasamos tres meses (hasta febrero 1801), sea en la casa del conde Orelly, sea en el campo, en casa del conde Jaruco y del marqués del Real Socorro. (...) Entonces me embarqué en Batabanó (Cuba); pero como por culpa de la incredulidad del piloto respecto a mis instrumentos, fuimos a dar al golfo del Darién, no llegamos a Cartagena, sino 35 días después (de otro modo la travesía dura apenas 14 días), el $1^{\circ}$ abril 1801 , no sin gran peligro (...).» ${ }^{36}$.

Como vemos, de nuevo le concede mucha más importancia a la travesía de Batabanó a Cartagena que a la descripción de sus actividades en la isla. También puede sorprender el hecho de que Humboldt, quien normalmente da informaciones, resultados de investigaciones $\mathrm{y}$, sobre todo, impresiones y criterios personales sobre los sitios que visita, referente a Cuba sólo brinda las noticias imprescindibles acerca de las fechas y de las personas en cuya casa estuvo.

Otra carta donde recoge una parte de sus investigaciones realizadas en la isla es la que dirige a Manuel de Espinosa y Tello desde México ${ }^{37}$. En ella le describe sus mediciones astronómicas y, detalladamente, los respectivos resultados de varios sitios, entre ellos diferentes lugares en el sur de Cuba.

Acerca de la segunda estancia de Humboldt en esta isla en la primavera de 1804, hemos podido localizar cuatro cartas enviadas desde allí. De la primera, enviada desde Güines a Jean Antoine Chaptal, lamentablemente sólo tenemos

\footnotetext{
34 Idem.

35 Carta escrita el 21 de septiembre de 1801 desde Contreras de Ibagué (Nueva Granada), en Minguet (1980), pp. 81-85.

36 Ibidem, p. 83.

37 Carta del 8.11.1803, en Moheit, U. (ed.), (1999), Das Gute und Grosse wollen. Alexander von Humboldts amerikanische Briefe, Berlin, Rohrwall Verlag, pp. 188-194.
} 
la referencia de que se recibiós ${ }^{38}$. Lo mismo pasa con una carta ${ }^{39}$ mandada desde La Habana a su hermano Wilhelm, de la que sabemos por un comentario que hace este último en otra misiva dirigida a Christiane von Haeften. Lo único que conocemos de su contenido - por lo que comenta Wilhelm - son los planes para la parte de la expedición que quedaba en este momento por realizar (estancia en los Estados Unidos y vuelta a Francia) ${ }^{40}$. También a Christiane von Haeften manda Alexander una carta desde La Habana, donde sólo se limita a expresarle su dolor por la muerte de Reinhard, el marido de aquélla, pero sin hacer comentarios sobre su estancia en $\mathrm{Cuba}^{41}$. La última carta encontrada la redactó para José de Espinosa y Tello, el hermano antes mencionado, y al igual que la dirigida a éste, aparte de una breve referencia al recorrido de su expedición contiene casi exclusivamente informaciones de tipo astronómico. En ella comenta Humboldt que en el año 1801 había realizado muchas observaciones de longitud y latitud desde Batabanó hasta Trinidad y en los Jardines de los Reyes, y le ofrece los resultados de estas mediciones, comparadas en parte con las observadas en México ${ }^{42}$.

Con su vuelta a Europa continúa con su costumbre de redactar largas cartas, llenas de impresiones, para informar a sus amigos de su regreso en buenas condiciones, pero que no contienen informaciones sobre su estancia en Cuba. Demasiado importante era para él el hecho de haber vuelto sano a Europa, trás una ausencia de cinco años. Por otra parte, había tenido demasiadas sensaciones en poco tiempo - su vuelta a Cuba, la estancia en Filadelfia y, finalmente, el término de su expedición con su regreso a Europa - para poder elaborar estas impresiones debidamente.

Como vemos, estas cartas no ofrecen informaciones relevantes sobre la primera y segunda estancias de Humboldt en Cuba, ni aclaran las dudas que surgen al estudiar las cuestiones tratadas en este trabajo. Así, por ejemplo, resulta significativo que en ninguna carta se encuentren sus impresiones personales sobre esta isla caribeña, ni tampoco —y es lo que más destaca- que no se refleje su opinión sobre el tema que tanto le ocupó durante su estancia allí: la esclavitud. Suponemos que Humboldt consideró inoportuno, o no recomendable, comentar estas reflexiones por cartas.

Resumiendo, se puede decir que es hasta cierto punto justificable la ausencia de descripciones en las cartas de Humboldt acerca de sus actividades en la

\footnotetext{
38 Carta del 27.3.1804, referencia en MoHeIT (1993), p. 279.

39 Con fecha del 28.3.1804.

40 Carta del 9.6.1804, en MoHeIt (1993), p. 284.

41 Del 27.3.1804, en ibidem, pp. 283-284.

42

Del 25.4.1804, en ibidem, pp. 286-290.
} 
isla de Cuba durante su segunda estancia. Habría estado muy ocupado con el envío de sus colecciones, la preparación de su visita a los Estados Unidos y la finalización de su gran expedición. Pero no deja de provocar asombro la falta de descripciones más detalladas o de sus impresiones personales sobre su primera visita en la isla, si se comparan con los largos relatos de su estancia en Venezuela.

\section{I.4. Memoria autobiográfica}

Humboldt da una visión bastante superficial de sus estancias en la isla caribeña - similar a la que brinda en otros textos oficiales (con excepción del Ensayo) - en una de las memorias autobiográficas que redactó a lo largo de su vida en distintas situaciones, el relato de su expedición americana que entrega a la American Philosophical Society de Filadelfia durante su visita a los Estados Unidos a finales de este viaje, en mayo y junio de $1804^{43}$.

Acerca de su primera estancia en Cuba se limita a comentar sus investigaciones científicas, a pesar de que, como sabemos, éstas no constituían allí su preocupación primordial.

«Humboldt permaneció tres meses en la isla de Cuba, donde se ocupó de medir la longitud de la Habana y de la construcción de hornos en los Ingenios, que después se han extendido mucho» ${ }^{44}$.

No es sorprendente que obvie el tema que tanto le había impactado en $\mathrm{Cu}-$ ba: la esclavitud, pues este resumen de su viaje estaba dirigido en primer lugar a los lectores de Estados Unidos.

A continuación hace énfasis —al igual que en las fuentes ya mencionadas - en las condiciones de su partida y en las mediciones realizadas:

«Humboldt salió de Batabanó en marzo de 1801, costeó el sur de la isla de $\mathrm{Cu}$ ba, donde determinó varias posiciones astronómicas. La falta de viento alargó mucho esta navegación, las corrientes llevaron la pequeña goleta demasiado al oeste hasta la embocadura del río Atrato» ${ }^{45}$.

\footnotetext{
43 ReBOK y PUIG-SAMPER (2002).

44 Ibidem, p. 77.

45 Idem.
} 
Las informaciones que nos facilita en este documento en relación con su segunda estancia son más rudimentarias todavía:

«Tras una corta estancia en Jalapa, por fin se embarcaron en Veracruz con rumbo a la Habana. Recogieron las colecciones que habían dejado en 1801 y tomaron la vía de Filadelfia para volver en julio de 1804 a Francia, después de 6 años de ausencia y de trabajos.» 46

En resumen, se puede constatar que la descripción que ofrece Humboldt en esta fuente no tiene mucho que ver con la realidad de sus estancias en Cuba y, por lo tanto, refleja más bien cómo él quería que se vieran en los Estados Unidos sus actividades realizadas en la isla.

II. INTERPRETACIÓN DE LA ESTANCIA DE HUMBOLDT EN CUBA A PARTIR DE SUS COMENTARIOS

Debido a lo escueto de las descripciones de Humboldt sobre sus estancias en Cuba, resulta algo difícil y especulativo reconstruir su recorrido por la isla. Como ya se dijo, tanto en sus escritos oficiales como en los no oficiales, concede relativamente más importancia a la llegada en barco a La Habana y a la salida desde Batabanó a Cartagena, que a la descripción de sus estancias en Cuba. Lo que más podría interesarnos, es decir, el tiempo que pasó en La Habana, sus actividades, contactos e impresiones, y sobre todo, sus opiniones sobre la sociedad esclavista en la que se encontró, no se hallan ni en sus cartas, ni en su narración del viaje, ni apenas en su diario. Soló en su Ensayo dedicó largos comentarios a la esclavitud y criticó duramente este sistema inhumano.

Por ello podemos preguntarnos: ¿qué es - aparte de lo dicho- lo que le interesó de Cuba, y especialmente de La Habana y sus alrededores? Lo que él mismo dice respecto a las investigaciones científicas que realizó durante su estancia.

Simplemente se pueden encontrar comentarios sueltos, más o menos extensos, sobre aspectos puntuales. Así, nos comenta que pasaron «los meses de diciembre, enero y febrero en hacer observaciones en las cercanías de la Habana y las hermosas llanuras de Güines» ${ }^{47}$; que allí tratan de reunir noticias

\footnotetext{
$46 \quad$ Ibidem, p. 82.

47 Humboldt (1998), p. 120.
} 
exactas acerca de los elementos numéricos de la fabricación del azúcar de caña ${ }^{48}$, y que en Güines y particularmente en Río Blanco, en casa del conde de Mopox, hizo «el ensayo de muchas construcciones nuevas, con el fin de disminuir el gasto del combustible, de rodear el hogar de substancias que conducen mal el calor, y conseguir que los esclavos sufriesen menos atizando el fuego» ${ }^{49}$. Nos comenta, además, en varias ocasiones sus observaciones astronómicas para averiguar la longitud de La Habana, que en aquella época estaba equivocada en más de $11 / 5$ de grado. Algunas informaciones más detalladas sobre sus viajes por Cuba y el carácter de sus actividades científicas - sobre todo lo que se refiere a su estudio de los cocodrilos y caimanes existentes en la Ciénaga de Batabanó- se encuentran en el ya mencionado último capítulo del Ensayo.

$\mathrm{Al}$ estudiar sus apuntes sobre Cuba, da la sensación que durante su estancia, Humboldt muestra relativamente menos interés por las investigaciones y mediciones que realiza, que por su participación en la vida social de la aristocracia cubana, y que asociaba estas estancias más bien con objetivos de carácter político, social y humanista que con fines científicos. La elaboración científica se llevó a cabo mucho después con la redacción de su Ensayo, si exceptuamos su recolección de plantas para editar la Flora Cubae Insulae, y la redacción de un breve escrito llamado Noticia mineralógica del cerro de Guanabacoa. Este último trabajo realizado durante su segunda estancia en La Habana, a petición de la habanera Sociedad Económica de Amigos del País que quería aprovecharse del talento y los conocimientos del célebre viajero prusiano, para que la informara sobre el valor minero de los cerros de Guanabacoa, próximos a esa ciudad. ${ }^{50}$

La reconstrucción de las ideas de Humboldt, 200 años después, inevitablemente da lugar a cierto carácter especulativo. En este caso se podría pensar que, antes de su primera llegada a Cuba, Humboldt había vivido en circunstancias muy sencillas en Venezuela - sobre todo durante su navegación en los ríos - y que ahora disfrutaba de condiciones acomodadas entre la aristocracia cubana, que con sus reuniones, cenas elegantes y tertulias de salón, en cierto sentido pueden haberle recordado la vida que había llevado antes de su expedición. También hay que tomar en cuenta que en Cuba, Humboldt podía presentarse por primera vez en sociedad como famoso viajero-explorador - antes en Madrid sólo tenía proyectos, ahora había realizado parte de ellos - y que te-

\footnotetext{
48 Ibidem, p. 225.

49 Ibidem, p. 240.

$50 \quad$ Ibidem, pp. 399-402.
} 
nía temas para entretener a la sociedad habanera; de esta manera se dejó integrar en las preocupaciones de la élite colonial cubana, que, como él mismo señala- consistían principalmente en la producción de azucar y la esclavitud. Esta sociedad esclavista - completamente opuesta a sus convicciones humanistas - le sorprendió tanto que le hizo ocuparse mentalmente de ese tema y de sus posibles soluciones.

En este contexto surge la pregunta: ¿porqué tardó Humboldt 22 años desde su vuelta a Europa en redactar su Ensayo sobre Cuba? Una posible explicación de carácter especulativo es que sus ideas sobre Cuba - basadas fundamentalmente en la abolición de la esclavitud y en una reforma del sistema económico y político - no podían ser publicadas de esta manera, si se quería evitar un conflicto tanto con el gobierno español como con la administración colonial de la isla. Según este criterio, sólo era factible una publicación después de obtener datos e informaciones complementarias de sus contactos y amigos en Cuba a lo largo de los años, para no limitar sus conclusiones a observaciones iniciales.

Comparando sus descripciones de tipo oficial con las no oficiales sobre Cuba, vemos con rapidez lo especial del caso del país caribeño. Contrasta considerablemente que incluya pocas informaciones en sus diarios y cartas respecto a la isla, y luego le dedique un libro entero; algo que no hace por ejemplo con Venezuela, que parece haberle llamado más la atención dada la cantidad de descripciones e informaciones detalladas que nos deja. Si queremos, por tanto, analizar las diferencias que se hallan en los escritos oficiales de Humboldt —en este caso la Relation historique y el Ensayo- y los escritos de carácter privado - sus cartas y diarios-, nos encontramos con el problema de la escasez de comentarios en sus apuntes no oficiales. Por esa razón dicho análisis no ha podido realizarse en la envergadura que hubiéramos deseado.

Lo que sí destaca a primera vista es que los juicios de Humboldt sobre la sociedad y el paisaje de Cuba, que aparecen en la Relation historique y el Ensayo - posteriormente redactados - se diferencian de manera considerable de la reacción espontánea y en parte bastante crítica de los diarios. En estos últimos Humboldt muestra, en general, una visión de Cuba más bien negativa; sus cartas, sin embargo, no aportan los juicios instantáneos y honestos respecto al tema, que se esperaba de esta fuente.

La explicación parece estar en su rechazo al sistema esclavista, además de la supuesta falta de inquietudes científicas de la élite colonial, como demuestran sus comentarios en los diarios, y el estado de desorden en que encontró La Habana. Analizando bien la Cuba de su época, se puede comprender per- 
fectamente la crítica social que recoge en sus diarios, así como la que hace a la administración colonial y a la organización de la agricultura. Sus comentarios negativos respecto al desarrollo de las ciencias, al contrario, pueden resultar algo sorprendentes, ya que indudablemente había una actividad científica en la Cuba de entonces - aunque posiblemente menor que en otras regiones de la América Española. A la vista está además, que su Ensayo sobre Cuba fue elaborado con datos e informaciones proporcionadas en parte por sus contactos cubanos, de lo que se deduce que sí había preocupaciones en este campo.

De todas maneras llama la atención que varios de los aspectos que critica de La Habana, seguramente no eran muy diferentes en otras capitales americanas que visitó, aunque no hiciera esta crítica de modo tan tajante. Tal postura sobre Cuba pudiera deberse a su rechazo profundo de la sociedad esclavista y capitalista que allí encontró, así como su preocupación por el desarrollo científico.

En su obra sobre México, parece reafirmar esta primera impresión:

«Son ciertamente muy notables estos progresos en Méjico, La Habana, Lima, Santa Fe, Quito, Popayán y Caracas. De todas estas grandes ciudades, La Habana se asemeja más a las de Europa en cuanto a sus costumbres, lujo refinado y tono de trato social. En La Habana se conoce mejor la situación de los negocios políticos y su influencia en el comercio. Con todo, a pesar de los esfuerzos de la Sociedad Patriótica de la isla de Cuba, que protege las ciencias con el más generoso celo, prosperan estas con lentitud; en un país donde el cultivo y precio de los productos coloniales reclaman toda la atención de sus habitantes» ${ }^{51}$.

Unos años más tarde, sin embargo, en su ensayo sobre Cuba de 1826, describe los cambios ocurridos en el panorama científico desde su estancia en la isla. En esa descripción menciona instituciones científicas que se habían creado ya en el siglo XVIII, lo que contrasta con la impresión recogida en sus diarios:

«La Sociedad Patriótica de La Habana (creada en 1793); las de Sancti-Spíritus, de Puerto Príncipe y de Trinidad, dependientes de ella; la universidad con sus cátedras de teología, de jurisprudencia, de medicina y de matemáticas, creadas desde el año de 1728, en el convento de Padres Predicadores, la cátedra de economía política, fundada en 1818; la de botánica agrícola, el museo y la escuela de anatomía descriptiva, dedica al celo ilustrado de don Alejandro Ramírez, la biblioteca pública, la escuela gratuita de dibujo y de pintura, la escuela náutica, las escuelas lancasterianas y el jardín botánico, son instituciones en parte nuevas y en parte antiguas, las unas

51 Humboldt, A. von (1991), Ensayo político sobre el Reino de Nueva España, Mexico, Ed. Porrúa, p. 79. 
son susceptibles y esperan mejoras progresivas, las otras una reforma total capaz de ponerlas en armonía con el espíritu del siglo y las necesidades de la sociedad ${ }^{52}$ ».

En su descripción de Cuba se manifiestan notables diferencias entre la impresión que nos transmite de la primera y de la segunda visita. Este contraste destaca sobre todo en sus reflexiones expresadas en los diarios, la fuente más auténtica para revelar su verdadero pensamiento. El factor importante para una percepción tan distinta de la misma región parece ser el punto de referencia que tiene en cada caso. Como ya hemos visto, Humboldt comenta que de su primera estancia en la isla - después de conocer la selva de Venezuelale había quedado una impresión bastante mejor que de la segunda estancia, donde comparó a Cuba con México en los aspectos científicos y culturales, quedando en desventaja la isla.

Analizando los estudios realizados hasta nuestros días sobre la estancia y las actividades de Humboldt en Cuba — sobre todo de Cuba, España y Alemanianos preguntamos, ¿cuáles son los temas y aspectos principales investigados y descritos en los numerosos trabajos sobre Humboldt y esta isla caribeña?

A primera vista vemos que los temas tratados o por lo menos mencionados en casi todas estas publicaciones son:

- La actitud de Humboldt sobre la esclavitud en Cuba y las valoraciones de carácter general referentes al humanismo de Humboldt.

- La prohibición de su obra en Cuba y la polémica por la traducción mutilada del Ensayo efectuada en 1856 por el esclavista y anexionista J. S. Thrasher53.

- Sus intereses por las haciendas azucareras (visita, informaciones para una mejora de esta industria).

- Los homenajes recibidos por Humboldt en Cuba.

Al contrario, los temas que quedan por tratar o profundizar todavía analizando, entre otras cosas, los posibles motivos por los que no han sido estudiados - podrían ser los siguientes:

- Un análisis de más profundidad de sus relaciones generales (colaboradores científicos y contactos personales) en Cuba y estudiar el papel que es-

52 HumboldT (1998), p. 214.

53 Una descripción detallada de estos hechos y las circunstancias políticas que llevaron a ello encontramos en BECK (1992), pp. 252-260. 
tas personas tenían en la Cuba de entonces, sus actividades, sus interconexiones etc. ${ }^{54}$.

- Un estudio crítico y de más profundidad de sus comentarios e informaciones reseñadas en los diarios.

- La cooperación entre Cuba y la RDA en la investigación humboldtiana ${ }^{55}$.

- Un detallado estudio de su recorrido por Cuba.

Resumiendo este análisis historiográfico de las estancias de Alexander von Humboldt en la isla de Cuba, nos enfrentamos con dos acontecimientos que a primera vista pueden parecer contradictorios.

Por un lado, en Cuba al explorador prusiano se le atribuye una gran fama como segundo descubridor y se destacan sus méritos como precursor de las ciencias e instituciones científicas. Esto contrasta con el hecho de que durante sus estancias aquí, Humboldt prestó poca atención a Cuba en sus escritos privados, es decir, en sus diarios y cartas. Incluso, como hemos observado anteriormente, en estos últimos se recogen opiniones bastante negativas respecto a la sociedad cubana. En la investigación humboldtiana han nacido diversas teorías sobre su aparente escaso interés por Cuba en el momento del viaje y que no parece haber apreciado realmente lo que vio en la isla antillana. Según estos planteamientos, el interés de Humboldt por este país caribeño, que demuestra con la dedicación de su famoso y detallado análisis político-social y económico, es posterior a la independencia de las demás colonias españolas en América y está relacionado con el significado que esta isla iba a tener para la metrópoli ${ }^{56}$.

Por otro lado, como hemos comentado en la introducción de este trabajo, es contrastante que Humboldt no escribiese mucho sobre sus actividades y

54 Para evitar algunos errores que siempre se repiten en la historiografía sobre su estancia en Cuba, por ejemplo el varias veces comentado encuentro con Alejandro O'Reilly no fue posible, ya que éste se muere antes de que Humboldt llegara a Cuba (1725-23.3.1794). Se trataba de su hijo Pedro Pablo O’Reilly.

55 Sería importante analizar en este contexto el papel de la RDA, para ver hasta qué punto en este proceso se puede constatar una influencia por parte de la república socialista de Alemania, ya que en estos años se realizaron muchas colaboraciones y proyectos de hermanamiento en la investigación humboldtiana. Un trabajo inicial que aborda estas relaciones es el de García GonZÁlez, A. (2002), «Reconocimientos y homenajes a la obra cubana de Alejandro de Humboldt», en GoMEZ (2002), pp. 207-225.

56 Ver argumentación en ZEUSKE, M. (2001, 2002), «Humboldt, Historismus, Humboldteanisierung. Der «Geschichtsschreiber von Amerika», die Massensklaverei und die Globalisierungen der Welt», Humboldt im Netz (http://www.uni-potsdam.de/u/romanistik/humboldt/hin), 3 y 4 , revista de internet, Berlin/Potsdam. 
estancias en Cuba, y en cambio le dedicase todo un tratado teórico y sociológico; algo que sólo realizó también con México, pero no con otras regiones visitadas por él durante un periodo más largo de tiempo.

$\mathrm{Al}$ querer encontrar explicaciones a estos hechos, nos encontramos ante la dificultad de reconstruir acontecimientos y reflexiones sucedidas hace dos siglos. Una parte de la investigación por lo tanto sólo puede basarse en especulaciones, sin tener mucha certeza de ello.

Por otra parte, los diarios de Humboldt, gracias a la edición de Margot Faak, ofrecen nuevos impulsos a la investigación humboldtiana y, en particular, dan un giro interesante al estudio de sus estancias en Cuba. Al integrar las reflexiones más privadas de Humboldt, redactadas en el momento que las vivió y no a la distancia de más de dos décadas, y sin la «censura» provocada por su deseo de no enfrentarse con el gobierno colonial español ni con sus contactos con la élite cubana, nos proporciona una imagen diferente $-\mathrm{O}$ tal vez más rica- de su impresión sobre esta isla de las Antillas que la que podemos construir sólo a partir de su narración del viaje y de su Ensayo. 